\title{
Tangential Sulcal Echoes Potential Pitfall in the Diagnosis of Parenchymal Lesions on Cranial Sonography
}

\author{
Richard A. Bowerman, MD
}

The sonographic appearance of normal neonatal cerebral sulci may mimic that of significant intracranial pathology. An echogenic pseudolesion can be seen when a sulcus is imaged through its long axis. Criteria are discussed for the differentiation of this normal variant from true parenchymal lesions with their attendant serious clinical implications. KEY wORDS: brain; neonate; pseudolesion; neurosonography; pitfall neonate; cranial sonography; intracranial hemorrhage; pitfall. ( $)$ Ultrasound Med 6:685, 1987).
$\mathrm{R}$ efinements in ultrasound technology continue to improve image quality and the ability to resolve normal anatomic structures and pathology within the neonatal brain. In many neonates, prominent echogenic areas may be identified within the cerebral hemispheres, similar in appearance to parenchymal lesions such as isolated intraparenchymal hemorrhage or periventricular leukomalacia (PVL). This mass-like pseudolesion appears when the scanning plane passes tangentially through a cerebral sulcus or peripheral hemispheric margin at the edge of a gyrus. It is important to recognize this normal variant so as not to mistake it for true pathology, which could result in further unwarranted workup or assignment of a potentially unfavorable prognosis to the patient.

\section{SONOGRAPHIC FINDINGS}

The echogenic sulcal pseudolesion is most often delineated on coronal imaging where the configuration of the brain is such that a large segment of a normal sulcus is

Received October 24, 1986, from the Department of Radiology, University of Michigan Medical Center, Ann Arbor, Michigan. Revised manuscript accepted for publication June 30, 1987.

Address correspondence and reprint requests to Dr. Bowerman: Dept. of Radiology, B1D502/0030, University of Michigan Hospital, 1500 E. Medical Center Drive, Ann Arbor, MI 48109-0030. imaged tangentially as it wraps around the margin of a gyrus (Fig. 1A). Continuous real-time scanning to the edges of the apparent abnormality will reveal its thin nature, which is confirmed by rotation of the transducer to an approximately orthogonal plane, where contiguity with the typical normal linear sulcal echoes is apparent (Fig. 1B).

\section{DISCUSSION}

Neurosonography is an established imaging modality for evaluating the neonatal brain. The most commonly diagnosed entity, intracranial hemorrhage, and the less frequently seen PVL, are both identified in their early evolution by a pronounced echogenic character replacing the more normally echopenic brain (Fig. 2). ${ }^{1-4}$ The tangential sulcal pseudolesion described above may strikingly resemble such real pathology, particularly if located deeply within the parenchyma.

Hypoxic/ischemic $(\mathrm{H} / \mathrm{I})$ lesions other than PVL may also present as echogenic parenchymal foci, either diffuse or discrete in distribution. ${ }^{5,6}$ Although the sulcal pseudolesion may resemble such focal disease, an H/I lesion may distort normal anatomic landmarks or lie deep within the parenchyma separate from the adjacent sulci (Fig. 3), in contrast to the sulcal pseudolesion which demonstrates contiguity with adjacent sulci and does

(ㄷ) 1987 by the American Institute of Ultrasound in Medicine $\bullet J$ Ultrasound Med 6:685-689, $1987 \bullet 0278-4297 / 87 / \$ 3.50$ 
Figure 1 A, sulcal pseudolesion. Mid coronal sonogram demonstrates a prominent echogenic mass (arrow) in the left parietal lobe superolateral to the body of the lateral ventricle (V). Note the absence on any normal sulci adjacent to the echogenic area except as separated by the full width of a gyrus (contrast with Fig. 3). B, atypical "parasagittal" scan steeply angled from the anteromedial to the posterolateral aspects of the left hemisphere, to intersect the anterior aspects of the ventricle as well as the more laterally positioned pseudolesion, confirms the thin anteroposterior dimension of the echogenic mass (arrowheads) and its contiguity with adjacent normal sulci (curved arrows), also of similar caliber. Straight arrows indicate approximate plane of section for Fig. 1A. $\mathrm{A}=$ anterior, $\mathrm{V}=$ ventricle.

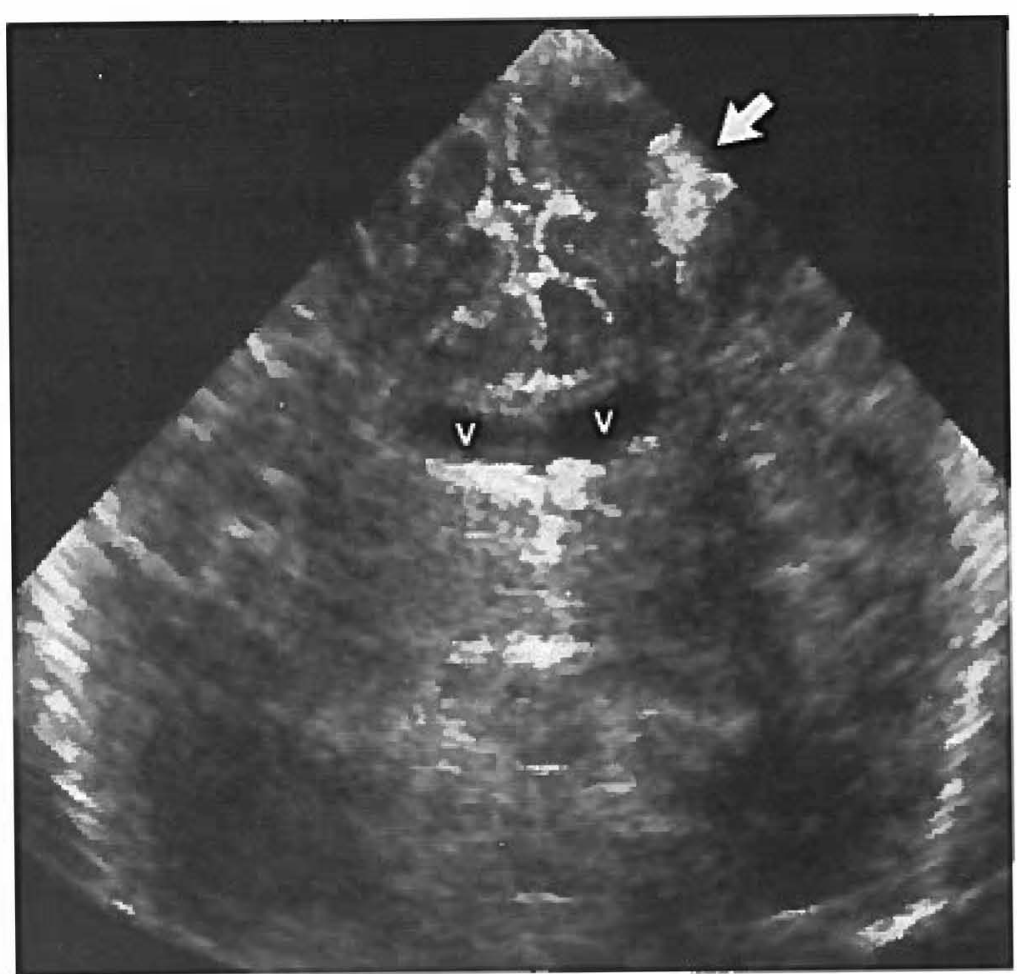

A

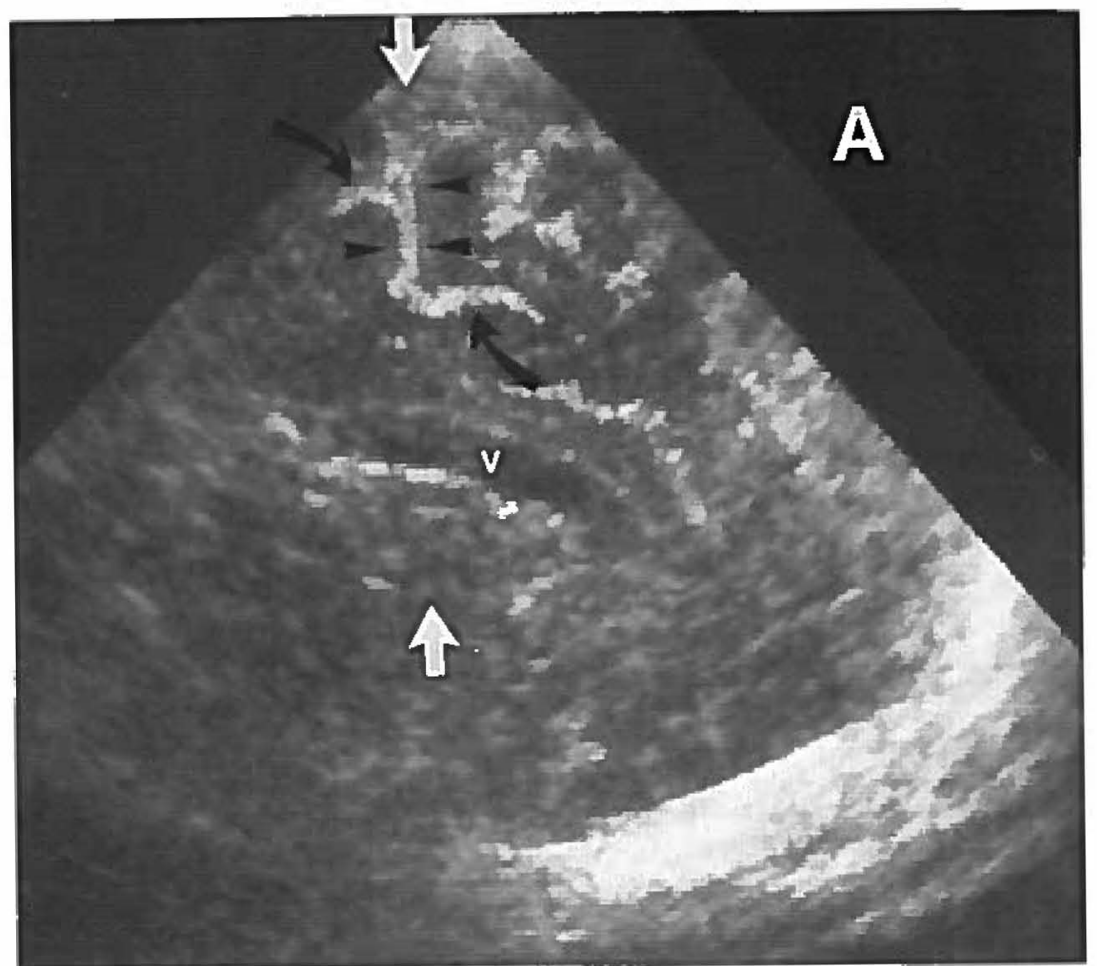




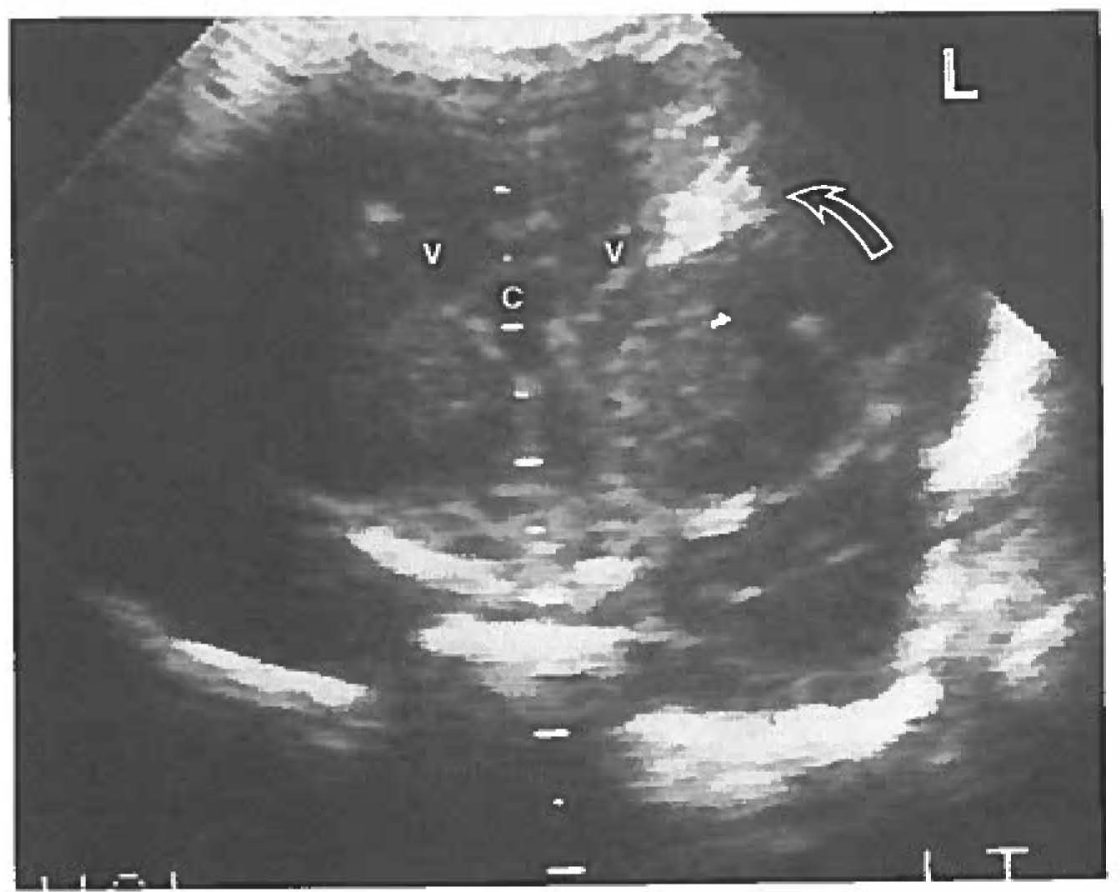

Figure 2 Periventricular leukomalacia. A, coronal and B, left parasagittal sonograms show an area of increased echogenicity in the left fronto-parietal distribution (arrows), superolateral to the lateral ventricle (V). In the coronal image, this is similar to the appearance of the echogenic sulcal pseudolesion; however, imaging in the orthogonal plane reveals the large anteroposterior dimension of the lesion as well. $\mathrm{L}=$ left, $P=$ posterior.

A

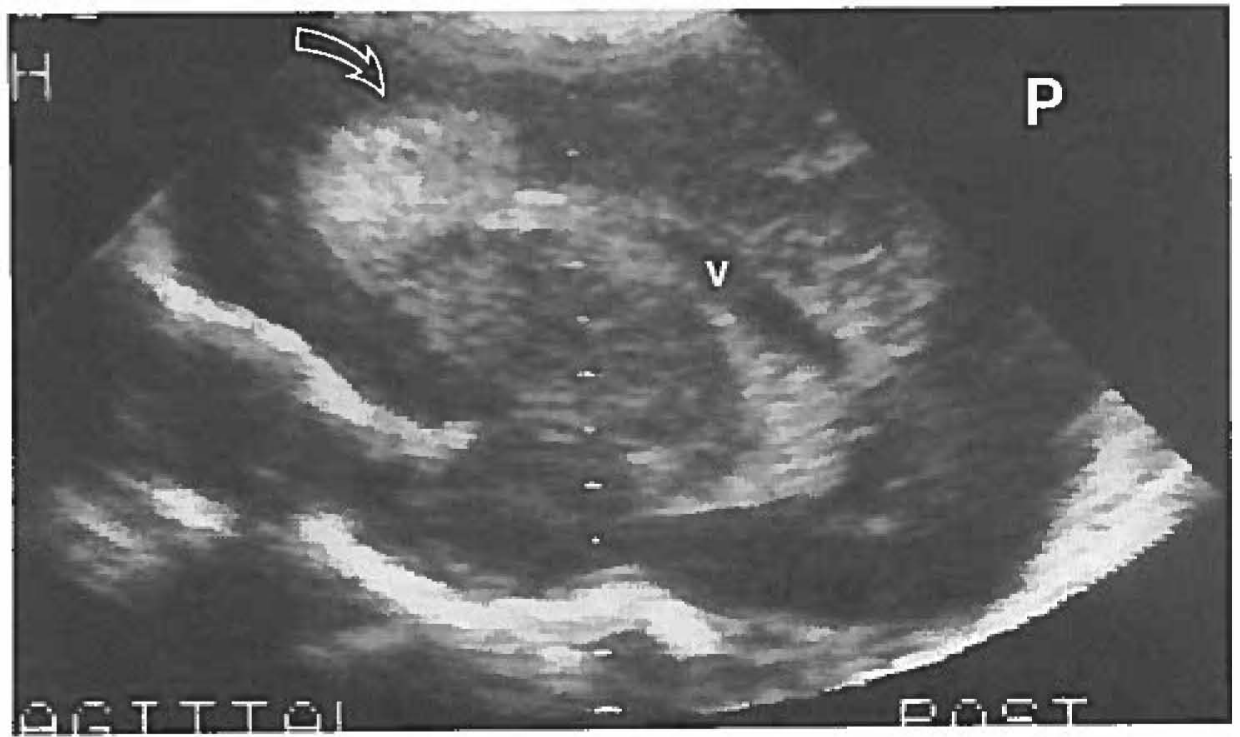

B

not alter normal structures.

Sulci appear in the developing fetal brain during the fifth month of gestation. ${ }^{7}$ By the 30 th week, the sparse, straight primary sulci start to bend and branch, secondary sulci appear, and the overall gyral pattern is developed such that the sulcal pseudolesion can be noted sonographically.

An inexperienced sonologist, particularly if reviewing static images from an earlier study, might well mis- interpret the sulcal pseudolesion as significant pathology. An awareness of this potential pitfall in the diagnosis of echogenic brain lesions should help one avoid an erroneous and potentially damaging diagnosis. Careful observation during actual scanning, or review of a videotaped exam, should confirm the discrepant thick/thin nature of the pseudolesion in orthogonal planes, the contiguity with normal sulci, and the lack of distortion of normal anatomic landmarks. 
Figure 3 Hypoxic/ischemic disease. Term infant with birth asphyxia secondary to meconium aspiration. A, angled mid coronal and B, far right parasagittal sonograms delineate abnormal foci of increased echogenicity within several gyri (arrowheads). Note the lack of contiguity with the adjacent sulci in two planes, in contrast to the sulcal pseudolesion. Computed tomography scanning confirmed multiple low attenuation areas consistent with edema and/or infarction. $\mathrm{V}=$ ventricle, $\mathrm{L}=$ left, $\mathrm{P}=$ posterior.

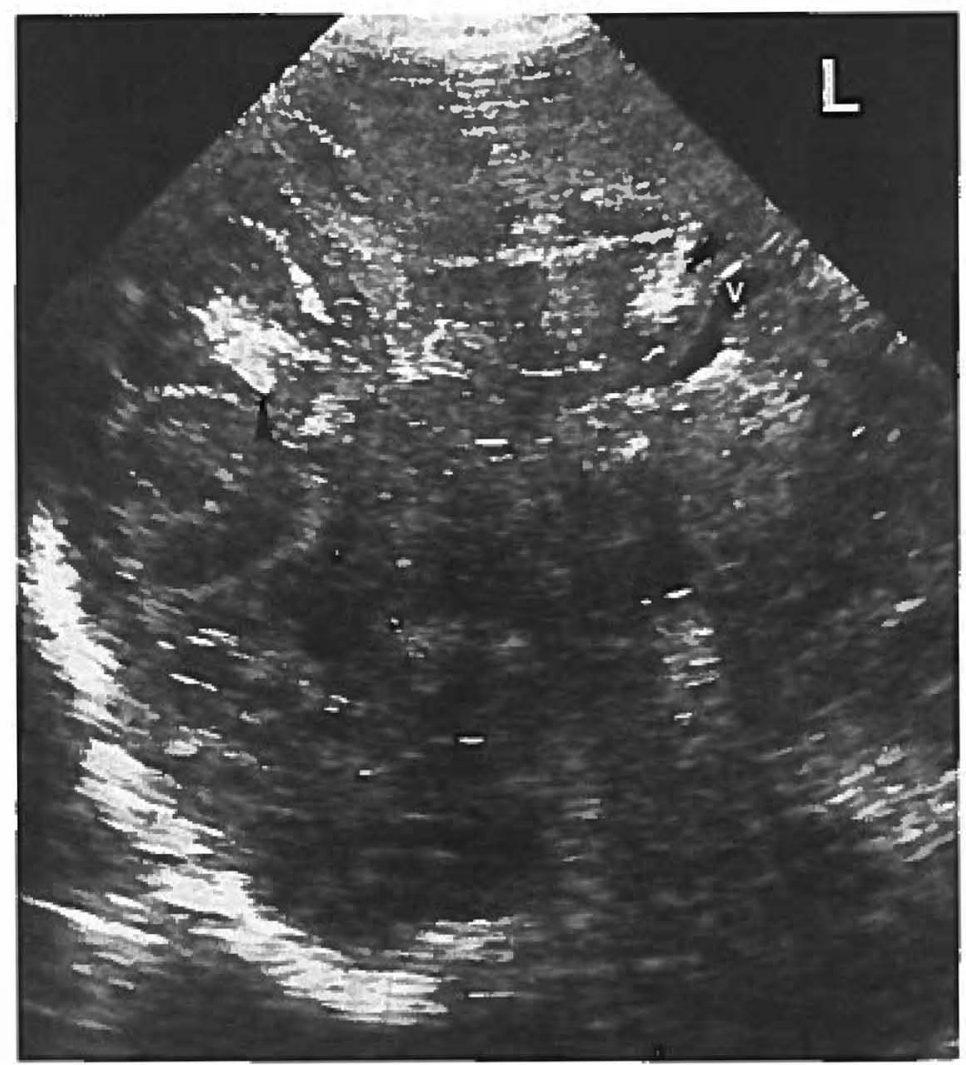

A






\section{REFERENCES}

1. Bowerman RA, Donn SM, Silver TM, et al: Natural history of neonatal periventricular/intraventricular hemorrhage and its complications: sonographic observations. AJR 143:1041, 1984 and AJNR 5:527, 1984

2. Slovis TL, Shankaran S: Ultrasound in the evaluation of hypoxic-ischemic injury and intracranial hemorrhage in neonates: the state of the art. Pediatr Radiol 14:67, 1984

3. Bowerman RA, Donn SM, DiPietro MA, et al: Periventricular leukomalacia in the pre-term infant: Sonographic and clinical features. Radiology 151:383, 1984
4. Schellinger D, Grant EG, Richardson JD: Cystic periventricular leukomalacia: sonographic and CT findings. AJNR 5:439, 1984

5. Babcock DS, Ball W, Jr: Postasphyxial encephalopathy in full-term infants: Ultrasound diagnosis. Radiology 148:417, 1983

6. Siegel MJ, Shackelford GD, Perlman JM, et al: Hypoxicischemic encephalopathy in term infants: diagnosis and prognosis evaluated by ultrasound. Radiology 152:395, 1984

7. Worthen NJ, Gilbertson V, Lau C: Cortical sulcal development seen on sonography: relationship to gestational parameters. J Ultrasound Med 5:153, 1986 